\title{
Is there a clinically relevant interaction between clarithromycin and statins not metabolized by cytochrome P450 3A4?
}

\author{
Daniel S. Streetman PharmD MS, Stephen M. Stout PharmD MS
}

See also research article on page 174 and at www.cmaj.ca/lookup/doi/10.1503/cmaj.140950

$\longrightarrow$ onsiderable data support the use of statins for the prevention of atherosclerotic cardiovascular diseases. ${ }^{1}$ An estimated 25 million patients worldwide are taking a statin, ${ }^{1,2}$ and according to the US National Health and Nutrition Examination Survey (2003-2012), more than $25 \%$ of American adults 40 years of age and older were taking a statin in 2011/12, an increase from about $18 \%$ in 2003/04. ${ }^{3}$ Statin use is expected to increase further, at least in part because of recently revised guidelines. ${ }^{1,4}$

Unfortunately, statin-related adverse effects limit the use of the drugs in many patients who might otherwise benefit from them. Most notable are muscle-related effects, varying from tolerable myalgia (estimated incidence of 190 per 100000 patient-years) to rhabdomyolysis (estimated incidence of 0.1 to 8.4 per 100000 patient-years). ${ }^{2}$ Increased statin concentration, due to higher doses or drug interactions, or both, is a wellknown yet modifiable risk factor for such effects.

In a linked research article, $\mathrm{Li}$ and colleagues ${ }^{5}$ report on adverse effects associated with statins not appreciably metabolized by cytochrome P450 3A4 (CYP3A4), and thus without clinically meaningful interactions with most strong CYP34A inhibitors. Specifically, they compare outcomes between patients taking one of the studied statins who were co-prescribed clarithromycin (a macrolide antibiotic that is a strong CYP34A inhibitor) or azithromycin (a macrolide antibiotic that does not inhibit CYP34A). Among those co-prescribed clarithromycin, they found a statistically significant increase in the risk of all-cause mortality and of hospital admission with acute kidney injury or hyperkalemia. The risk of admission with rhabdomyolysis was not significantly increased.

One strength of the study is the large number of patients in the population-based cohort. Such a large sample is critical when attempting to evaluate uncommon outcomes such as rhabdomyolysis. The authors have also done a commendable job working to address the limitations inherent to a study of this design. Most notably, in a prior publication, the authors compared patients taking clarithromycin with those taking azithromycin to determine the comparability of such patients for population-based studies investigating potential drug interactions. ${ }^{6}$ The two groups were largely similar in demographic characteristics and outcomes; however, data regarding the cause of infection were missing for more than $50 \%$ of the patients, and clarithromycin treatment was associated with a significant increase in all-cause mortality (adjusted relative risk $1.27,95 \%$ confidence interval 1.04-1.55).

Despite its many strengths, the current study has some limitations that merit attention. First, the reliance on diagnostic codes to identify the outcomes of interest may not only underestimate total risk, as the authors acknowledge, but also distort relations in the data by not differentiating between attributable and nonattributable outcomes. In a separate population-based study involving 292 patients with an International Classification of Diseases ninth revision (ICD-9) code for rhabdomyolysis, only 22 (7.5\%) were validated as having statin-related rhabdomyolysis upon full review of the electronic medical records. ${ }^{7}$ This also led to different estimates of the risk of high- versus lower-dose simvastatin, with an incidence rate ratio (IRR) of 1.77 in the ICD-9-defined cohort and an IRR of 12.2 in the validated cohort. That the median creatine kinase concentration was only $1835 \mathrm{U} / \mathrm{L}$ among patients with an ICD-10 code for rhabdomyolysis in Ontario (an observation stated by Li and col-
Competing interests: None declared.

This article was solicited and has not been peer reviewed.

Correspondence to: Daniel Streetman, daniel.streetman @ wolterskluwer.com

CMAJ 2015. DOI:10.1503 /cmaj.150030 
leagues from unpublished data) raises concerns about misclassification in the current study: at least half of the patients identified as having rhabdomyolysis would apparently not meet commonly applied criteria (i.e., creatine kinase level $\geq 10$ times the upper limit of normal).

Second, Li and colleagues excluded patients who were taking strong CYP3A4 inhibitors, out of concern that such drugs also commonly inhibit organic anion-transporting polypeptide 1B1 (OATP1B1). Although some strong CYP3A4 inhibitors may also inhibit OATP1B1, no substantial evidence of a correlation between CYP3A4 inhibition and OATP inhibition is offered in the cited references or, to our knowledge, in other publications. Conversely, the authors did not exclude patients who were taking some betterestablished OATP1B1 inhibitors, such as gemfibrozil or rifampin. Also, it is unclear whether moderate inhibitors of CYP3A4, such as cyclosporine, were excluded. Because only small numbers of patients using CYP3A4 inhibitors were excluded, and because use of other inhibitors would not be expected to differ between the study groups, the impact of the authors' decision to exclude potential OATP1B1 inhibitors is questionable. However, with the small numbers of outcomes, differences in use of other OATP1B1 inhibitors could be meaningful.

Ultimately, how should these findings affect practice? The degree to which the observed risks are attributable to a clarithromycin-statin interaction remains uncertain. Specifically, acute kidney injury and hyperkalemia would be unusual signs of statin toxicity in the absence of rhabdomyolysis, which did not differ significantly between the study groups. Also, the reported 30-day absolute and relative mortality rates associated with clarithromycin and azithromycin are similar to those reported without concurrent statin use. ${ }^{6}$ Even if fully attributed to an interaction, the differences in absolute risk are so small that more than 900 to 3000 patients would need to be exposed to these interactions to result in 1 additional hospital admission because of acute kidney injury or hyperkalemia, or death.

With the volume of statin use, avoiding harmful outcomes with even this low an incidence may have a substantial population benefit. However, non-CYP3A4-metabolized statins account for a minority of all statin use, ${ }^{3}$ and because these estimates were derived from an older population (mean age $>73 \mathrm{yr}$ ), actual risks would likely be lower in the broader population taking statins. Further, better-established statin interactions often remain overlooked in practice, as highlighted by a recent review of claims data that found use of simvastatin in doses exceeding those recom- mended during treatment with certain calciumchannel blockers dropped modestly from $60 \%$ to $41 \%$ in the nine months following addition of the dose limits to simvastatin product labelling. ${ }^{8}$ Many patients also continued to receive agents newly listed as contraindicated with simvastatin, particularly gemfibrozil. This challenge of influencing practice casts additional doubt on the prospective population benefit of informing clinicians about interactions with low risk.

Hopefully, the findings from the current study will alert clinicians to the reality that many factors beyond CYP3A4 inhibition influence systemic statin concentrations and related adverse effects. Liver-specific OATP1B1, which the authors suggest as a mediator of the proposed interaction between clarithromycin and nonCYP3A4-metabolized statins, does appear to control hepatic uptake of many statins at least partially. Consequently, decreased activity or expression of OATP1B1, whether due to drug interactions, genetic variation or other factors, would be expected to result in diminished hepatic uptake and decreased elimination of most statins (predisposing to adverse effects and decreased effectiveness).

Importantly, OATP1B1-mediated uptake does not appear to be equally important to all statins. In individuals who were homozygous for a lower-activity variant in the SLCO1B1 gene that codes for OATP1B1, increases in concentrations of simvastatin were almost twice as much as increases in concentrations of pravastatin and rosuvastatin $(221 \%$ increase for simvastatin v. $57 \%-130 \%$ increase for pravastatin and 62\%$117 \%$ increase for rosuvastatin). ${ }^{9,10}$ Concentrations of fluvastatin were not significantly higher in these patients. Similarly, drugs widely considered to be OATP1B1 inhibitors have varying magnitudes of interaction with statins. For example, concurrent gemfibrozil use (which also appears to inhibit CYP2C8 and some glucuronidation pathways) has been associated with twoto threefold increases in pravastatin, rosuvastatin, simvastatin and lovastatin concentrations; however, it was associated with only a $35 \%-45 \%$ increase in atorvastatin and pitavastatin concentrations, and no significant change in fluvastatin concentrations. In addition to CYP3A4 and OATP1B1, the disposition of individual statins has been associated with several other drugmetabolizing enzymes (e.g., CYP3A5, CYP2C8 and CYP2C9) as well as other drug transporters (e.g., P-glycoprotein and sodium taurocholate cotransporting polypeptide [NTCP]), which further underscores both the complex nature of statin metabolism and elimination and the difficulties in predicting risks of drug interaction. 


\section{References}

1. Stone NJ, Robinson JG, Lichtenstein AH, et al.; American College of Cardiology/American Heart Association Task Force on Practice Guidelines. 2013 ACC/AHA guideline on the treatment of blood cholesterol to reduce atherosclerotic cardiovascular risk in adults: a report of the American College of Cardiology/American Heart Association Task Force on Practice Guidelines. J Am Coll Cardiol 2014;63(25 Pt B):2889-934.

2. Alfirevic A, Neely D, Armitage J, et al. Phenotype standardization for statin-induced myotoxicity. Clin Pharmacol Ther 2014; 96:470-6.

3. Gu Q, Paulose-Ram R, Burt VL, et al. Prescription cholesterollowering medication use in adults aged 40 and over: United States, 2003-2012 [NCHS data brief no. 177]. Hyattsville (MD): National Center for Health Statistics; 2014.

4. Pencina MJ, Navar-Boggan AM, D'Agostino RB Sr, et al. Application of new cholesterol guidelines to a populationbased sample. N Engl J Med 2014;370:1422-31.

5. Li DQ, Kim R, McArthur E, et al. Risk of adverse events among older adults following co-prescription of clarithromycin and statins not metabolized by cytochrome P450 3A4. CMAJ 2014; Dec. 22. [Epub ahead of print].

6. Fleet JL, Shariff SZ, Bailey DG, et al. Comparing two types of macrolide antibiotics for the purpose of assessing populationbased drug interactions. BMJ Open 2013;3:e002857.

7. Floyd JS, Heckbert SR, Weiss NS, et al. Use of administrative data to estimate the incidence of statin-related rhabdomyolysis. JAMA 2012;307:1580-2.

8. Tuchscherer RM, Nair K, Ghushchyan V, et al. Simvastatin prescribing patterns before and after FDA dosing restrictions: a retrospective analysis of a large healthcare claims database. Am J Cardiovasc Drugs 2014 Oct 28. [Epub ahead of print].

9. Kalliokoski A, Niemi M. Impact of OATP transporters on pharmacokinetics. Br J Pharmacol 2009;158:693-705.

10. Ramsey LB, Johnson SG, Caudle KE, et al. The Clinical Pharmacogenetics Implementation Consortium guideline for SLCO1B1 and simvastatin-induced myopathy: 2014 update. Clin Pharmacol Ther 2014;96:423-8.

Affiliation: Metabolism, Interactions and Genomics Group, Wolters Kluwer Health, Hudson, Ohio

Contributors: Both authors contributed substantially to the drafting and revising of the manuscript, approved the final version submitted for publication and agreed to act as guarantors of the work 